Western University

Scholarship@Western

Chemistry Publications

Chemistry Department

2015

\title{
Tunable 3D Plasmonic Cavity Nanosensors for Surface-enhanced Raman Spectroscopy with Sub Femtomolar Limit of Detection
}

Mohammadali Tabatabei

Western University

Mohamadreza Najiminaini

Western University

Kiefer Davieau

Western University of Health Sciences

Bozena Kaminska

Simon Fraser University

Mahi Singh

Western University

See next page for additional authors

Follow this and additional works at: https://ir.lib.uwo.ca/chempub

Part of the Chemistry Commons

Citation of this paper:

Tabatabei, Mohammadali; Najiminaini, Mohamadreza; Davieau, Kiefer; Kaminska, Bozena; Singh, Mahi; Carson, Jeff; and LagugneLabarthet, Francois, "Tunable 3D Plasmonic Cavity Nanosensors for Surface-enhanced Raman Spectroscopy with Sub Femtomolar Limit of Detection" (2015). Chemistry Publications. 116.

https://ir.lib.uwo.ca/chempub/116 
Authors

Mohammadali Tabatabei, Mohamadreza Najiminaini, Kiefer Davieau, Bozena Kaminska, Mahi Singh, Jeff Carson, and Francois Lagugne-Labarthet 


\section{Tunable 3D plasmonic cavity nanosensors for}

2 surface-enhanced Raman spectroscopy with sub-

8 2Imaging Program, Lawson Health Research Institute St. Joseph's Health Care, London, ON,

$$
\text { Western Ontario, London, ON, N6A 5C1, Canada }
$$

${ }^{4}$ The School of Engineering Science, Simon Fraser University, Burnaby, BC, V5A 1S6, Canada

15 Corresponding Authors

16 *E-mail: flagugne@uwo.ca (F.L.-L.); jcarson@lawsonimaging.ca (J. J. L. C.) 


\section{ABSTRACT}

18 Metallic nanohole arrays (NHAs) with a high hole density have emerged with potential

19 applications for surface-enhanced Raman spectroscopy (SERS) including the detection of 20 analytes at ultra-low concentrations. However, these NHA structures generally yield weak

21 localized surface plasmon resonance (LSPR) which is a prerequisite for SERS measurements. In

22 this work, a compact three-dimensional (3D) tunable plasmonic cavity with extraordinary optical

23 transmission properties serves as a molecular sensor with sub-femtomolar detection. The 3D

24 nanosensor consists of a gold film containing a NHA with an underlying cavity and a gold 25 nanocone array at the bottom of the cavity. These nanosensors provide remarkable surface 26 plasmon polariton (SPP) and LSPR coupling resulting in a significantly improved detection 27 performance. The plasmonic tunability is evaluated both experimentally and theoretically. A 28 SERS limit of detection of $10^{-16} \mathrm{M}$ for 4-Nitrothiophenol (4-NTP) is obtained along with 29 distribution mapping of the molecule on the 3D plasmonic nanosensor. This results in an 30 improved SERS enhancement factor (EF) of $10^{6}$ obtained from a femtolitre plasmonic cavity 31 volume. The tunability of these sensors can give rise to a potential opportunity for use in optical 32 trapping while providing SERS sensing of a molecule of interest.

33 KEYWORDS: Surface-enhanced Raman spectroscopy (SERS), 3D nanosensor, Plasmonics, 34 Surface plasmon polariton (SPP), Localized surface plasmon resonance (LSPR), Sub-femtomolar 35 limit of detection. 


\section{INTRODUCTION}

38 Molecular plasmonics has shown great promise for trace detection of molecules and

39 biomolecules adsorbed onto rationally designed metallic platforms or particles. ${ }^{1-4}$ Among the

40 techniques benefiting from recent advances of plasmon-mediated optical measurements, surface-

41 enhanced Raman spectroscopy (SERS) has pushed the limits for detection of even lower

42 concentration of analytes. It yields chemical and biological sensing using a variety of surfaces

43 and approaches that mainly relies on the drastic enhancement of the weak Raman signal through

44 the enhancement of the local electromagnetic field in the vicinity of the metallic surface. ${ }^{5-6}$ As an

45 analytical technique, SERS provides label-free sensing with high sensitivity and chemical

46 specificity. ${ }^{7-8}$ Rational development of SERS platforms includes the production of reproducible

47 engineered metallic platforms with arrays of well-defined structures that combine the functions

48 of (i) trapping the analyte of interest, (ii) improving the sensitivity of the measurement by several

49 orders of magnitude and (iii) providing quantitative measurements. As the enhancement of

50 electromagnetic fields occurs in nanoscale regions, so-called plasmonic hot spots, integration of

51 specific nanostructures for trapping the interested molecule in these hot spots can improve the

52 sensitivity and reduce the detection time for SERS. ${ }^{9-13}$

53 Arrays of periodic nanoholes in a metallic film can act as a plasmonic substrate with diverse

54 applications. ${ }^{14-17}$ The nanohole array (NHA) structure in an optically thick metal film allows for

55 momentum matching between an incident light on a NHA and the surface plasmon (SP) waves

56 existing at the interface between a metal and a dielectric material. ${ }^{15}$ The excitation of SPs, so-

57 called surface plasmon polariton (SPP), by light incident on a metallic NHA results in

58 extraordinary optical transmission (EOT) and optical resonances. The EOT properties of a NHA 
59 depend greatly on material composition and geometrical parameters of the structure. ${ }^{18-21}$ Owing

60 to scattering order of nanoholes, spacing between them and variable angle of incidence on the

61 NHA, various EOTs related to different SPP modes can be generated at different optical

62 frequencies such as $(1,0)$ and $(1,1)$ transmission resonances. ${ }^{15}$ NHAs have been widely used in

63 various plasmonic applications ranging from optical trapping to sensing of biologically relevant

64 molecules. $^{20,22-28}$ For instance, the array acts like a tunable filter since the wavelength selectivity

65 of the array transmission can be adjusted simply by changing the periodicity. ${ }^{29-30}$ Many studies

66 have been performed to improve the performance of NHAs for sensing applications. For

67 example, one of the most common applications of NHAs is surface plasmon resonance (SPR)

68 refractive index sensing. ${ }^{22,} 25,31$ It has been shown that the most optimal performance was

69 achieved in SPR sensing for a NHA structure consisting of an ultra-smooth NHA metal surface,

70 elliptical nanohole shapes, and nearly SP energy matching between the top and bottom surfaces

71 of the NHA. ${ }^{32}$

72 A localized surface plasmon resonance (LSPR) occurs when the incident light interacts with

73 surface plasmon confined in the vicinity of a metallic nanoparticle, the size of which being

74 comparable to or smaller than the excitation wavelength. ${ }^{33-35}$ As a result, the electromagnetic

75 field located in the near-field of the surface is greatly enhanced. ${ }^{36-37}$ In this context, the aim is to

76 design a plasmonic structure that will generate a strong LSPR coupling to further enhance the

77 signal of the adsorbed molecule on a 2D or 3D plasmonic nanostructure. ${ }^{38}$ Such LSPR plays a

78 pivotal role in many surface-enhanced spectroscopic techniques such as surface-enhanced

79 fluorescence, Raman and infrared spectroscopies, so-called SEF, SERS, and SEIRA,

80 respectively. ${ }^{33-34,39}$ 
81 An individual nanohole can produce transmission based LSPR, which is associated to an

82 interaction of the incident light with a LSPR around the nanohole. ${ }^{29}$ The similar properties have

83 been reported for a NHA with enhanced electric field intensity around each nanohole at the

84 corresponding resonance wavelengths. ${ }^{23}$ For SERS measurements, NHAs provide typical EFs

85 below $10^{5}$ which are weaker than other reliable SERS substrates. ${ }^{11-12,40}$ More importantly, these

86 EFs are not reported for very low concentrations of the probe molecules. The reported range is

87 between $2-10^{4}$, which is still comparable with some SERS substrates but not likely reliable

88 compared to sensitive SERS substrates with EFs of over $10^{5}{ }^{8,12,41}$

89 Herein, we evaluated the SERS performance of a 3D metallic nanostructure composed of an

90 array of nanoholes and co-registered nanocones embedded in a single cavity. The optical

91 properties of the nanosensors were investigated experimentally and using optical field modeling.

92 Three major features of these sensors are highlighted in this work: i) plasmonic tunability; ii)

93 surface enhanced Raman spectroscopy (SERS) of 4-Nitrothiophenol (4-NTP) covalently attached

94 on these sensors, and finally iii) limit of detection of 4-NTP adsorbed onto the sensors with fast

95 acquisition time along with mapping the distribution of the molecules over the platform

96 generating strong signals on the sensors based on the molecular fingerprint.

97 RESULTS AND DISCUSSION

98 Physical characterization of the fabricated 3D plasmonic cavity nanosensors

99 Scanning electron microscopy (SEM) images of the nanosensors are shown in Figure 1. These 100 sensors are composed of a NHA membrane with co-registered nanocone array (NCA). As shown 101 in Figure 1a, this NHA-NCA platform consists of a NHA membrane in a $230 \mathrm{~nm}$ thick Au film 
102 on a Pyrex substrate with a $250 \mathrm{~nm}$ deep cavity, below the surface of the Au film. It can be seen

103 in Figure $1 \mathrm{~b}$ that at the bottom of the cavity, the co-registered truncated nanocones are aligned

104 with the center of the nanoholes. The schematic representation of 3D plasmonic cavity

105 nanosensors is shown in Figure SI.1a. Demonstrated in Figure 1b, a truncated cone has a height

106 of $150 \mathrm{~nm}$ with an apex $100 \mathrm{~nm}$ away from the Au film. The hole sizes varied from 74 to $87 \mathrm{~nm}$

107 and the periodicities varied from 425 to $500 \mathrm{~nm}$ with increments of $25 \mathrm{~nm}$. A nanohole consists

108 of two truncated nanocones with their apices connected at the center of the nanohole. The apex

109 of the cone has a 1:2 ratio with respect to the cone base diameter. For the simulations, the

110 complex refractive indices of $\mathrm{Au}$ were provided by Palik and a refractive index of 1.474 was

111 used for Pyrex. ${ }^{42}$

\section{Tunable cavity 3D nanosensors}

113 In both simulated and experimentally measured optical transmission spectra of the 3D

114 nanosensors in Figure 2, multiple transmission resonances were observed due to the SPP

115 corresponding to various scattering mode indices. A metallic NHA with a square lattice

116 arrangement of nanoholes results in momentum matching between the in-plane wave-vectors of

117 the incident light and the SP, when $\overrightarrow{\mathrm{k}}_{\mathrm{sp}}=\frac{\omega}{c} \sin \theta \pm m \overrightarrow{\mathrm{u}}_{\mathrm{x}} \pm n \overrightarrow{\mathrm{u}}_{\mathrm{y}}$ is satisfied. The expression

$118 \frac{\omega}{c} \sin \theta$ is the in-plane component of the wave vector of the incident light, where $\omega$ is the

119 frequency of the incident light, $\mathrm{c}$ is the speed of the light, and $\theta$ is the incident angle of light. ${ }^{43}$

120 The reciprocal lattice wave vectors $\overrightarrow{\mathrm{u}}_{\mathrm{x}}$ and $\overrightarrow{\mathrm{u}}_{\mathrm{y}}$ describe a square lattice when $\left|\overrightarrow{\mathrm{u}}_{\mathrm{x}}\right|=\left|\overrightarrow{\mathrm{u}}_{\mathrm{y}}\right|=\frac{2 \pi}{a}$,

121 where $a$ is the spacing between adjacent nanoholes, and $\mathrm{m}$ and $\mathrm{n}$ are integers expressing the

122 scattering mode indices. From the conservation of energy, the SP dispersion relationship on a 
123 smooth metal surface can be expressed as $\left|\overrightarrow{\mathrm{k}}_{\mathrm{sp}}\right|=\left|\overrightarrow{\mathrm{k}}_{0}\right| \sqrt{\frac{\varepsilon_{\mathrm{m}} \varepsilon_{\mathrm{d}}}{\varepsilon_{\mathrm{m}}+\varepsilon_{\mathrm{d}}}}$, where $\varepsilon_{d}$ and $\varepsilon_{m}$ are the

124 dielectric functions of the incident medium (at the top or bottom surface of nano-hole) and the

125 metal film. By combining the momentum matching condition of the light-SP for light at normal

126 incidence to the NHA and the dispersion relation of the SP, the EOT positions of a NHA

127 associated to the SPP can be expressed by Equation 1:

$$
\lambda_{\max } \cong \frac{a}{\sqrt{m^{2}+n^{2}}} \sqrt{\frac{\varepsilon_{m} \varepsilon_{d}}{\varepsilon_{m}+\varepsilon_{d}}}
$$

129 The simulated and experimentally measured optical transmission spectra of the platforms for 130 various periodicities are shown in Figure 2 for platforms surrounded by air $(\mathrm{n}=1.00)$ or 131 immersed in water $(\mathrm{n}=1.33)$. When the structures are in air, both simulation and experimental 132 results showed a single transmission resonance, which can be associated to the $(-1,0)$ excitation 133 of the SP on the top and bottom surface of the NHA membrane. However, this resonance has 134 been also induced with the presence of truncated nanocone at the bottom of the cavity. The apex 135 of the truncated nanocone and the bottom of the nanohole would generate nanoantenna effect at 136 the resonance wavelength, which would generate localized SP between two afore-mentioned 137 nanosensors. As a result, $(-1,0)$ resonance is related to not only SPPs but also LSPRs. As the 138 periodicity of the hole decreases, the resonance transmission of the 3D nanosensors are blue139 shifted to shorter wavelengths (Figure 2a and c). The existence of LSPR coupling was observed 140 between the nanocone and nanohole in the simulation model, which resulted in generation of an 141 antenna and strong hot spot within this area. Although a nanocone structure without the presence 142 of nanohole could have two LSPR absorption resonances (at base and at apex), the combination 
144 the bottom surface of its corresponding nanohole.

145 The presence of a nanocone decays resonance transmission of a NHA due to the shadowing

146 effect and optical absorption of the nanocone. ${ }^{23}$ However, a NHA without nanocones cannot

147 generate strong LSPR similar to the proposed structure and would have more limited detection

148 and sensitivity in the SERS applications according to the previous studies on NHAs. ${ }^{11-12,40}$

149 Due to the bulk plasmon wavelength of $\mathrm{Au}$ at $500 \mathrm{~nm}$, the resonance of the 3D nanosensors

150 decays for smaller periodicity as the resonance blue-shifts towards $500 \mathrm{~nm}$. When the 3D

151 nanostructure is encapsulated in water, the LSPR-SPP mediated resonances of the 3D

152 nanosensors are red-shifted towards longer wavelengths (Figure $2 b$ and $d$ ). Two LSPR-SPP

153 resonances were seen in the optical transmission spectra of the 3D nanosensors. The resonances

154 were associated to $(-1,0)$ and $(1,1)$ scattering hole orders of the 3D nanosensors. The electric

155 field intensity of 3D nanosensors with $500 \mathrm{~nm}$ periodicty is shown in Figure 3 at the LSPR-SPP

156 resonance wavelengths of 633 and $785 \mathrm{~nm}$ for both air and water surrounding media. The electric

157 field distributions at LSPR-SPP resonances for air and water confirm that there is a strong LSPR

158 coupling between the bottom of the hole and the apex of the nanocone. This structure generates

159 the highest electric field at the resonance peak. However, the electric field at the $(1,1)$ LSPR-

160 SPP resonance is of weaker intensity compared to the electric field at $(-1,0)$ resonance. The

161 electric field at $633 \mathrm{~nm}$ appeared to be more intense in air, whereas it was lower when the

162 platform was immersed in water. This was due to the presence of the $(-1,0)$ resonance peak close

163 to $633 \mathrm{~nm}$ for a 3D nanosensor with $500 \mathrm{~nm}$ periodicity located in air. In contrast, the electric

164 field at $785 \mathrm{~nm}$ was significantly higher within the apex of the truncated cone and the bottom 
165 surface of the hole compared to that obtained in air at $785 \mathrm{~nm}$. There was a high absorption at the

166 base of the truncated nanocone due to the LSPR absorption properties of the 3D nanosensor.

167 Similar results are expected when the platform is immersed in a polar organic solvent such as

168 ethanol $(\mathrm{n}=1.36)$ based on its simulated optical transmission shown in Figure SI2.

169 Effect of plasmonic tunability on SERS

170 As shown in Figure 4., the SERS spectra of the 4-NTP molecules (1 mM) were collected on

171 different 3D nanosensors in two different media (air and water) and also for two wavelengths of

172 incident light (633 and $785 \mathrm{~nm}$ ). Using the $633 \mathrm{~nm}$ laser, the SERS signals of 4-NTP integrated

173 for the $v_{\mathrm{s}} \mathrm{NO}_{2}$ mode $\left(1337 \mathrm{~cm}^{-1}\right)$ were stronger for 3D nanosensors with periodicities of 500

$174\left(3.2 \times 10^{4}\right.$ a.u. $)$ and $425 \mathrm{~nm}\left(2.5 \times 10^{4}\right.$ a. u. $)$ when the signal was collected in air compared to

175 water (Figure 4a and b). When the $785 \mathrm{~nm}$ laser was used, the SERS intensities of 4-NTP were

176 stronger for both 3D nanosensors, P500 $\left(5.4 \times 10^{3}\right.$ a. u. $)$ and P425 $\left(4.1 \times 10^{3}\right.$ a. u. $)$ when collected

177 in water compared to air (Figure $4 \mathrm{c}$ and $\mathrm{d}$ ). These phenomena are also related to the tuned 178 plasmonic bands of the 3D nanosensors in air and water as shown in Figure 2. We observed 179 similar responses for 3D nanosensors with periodicities of $475 \mathrm{~nm}$ and $450 \mathrm{~nm}$ (Figure SI3). We

180 also observed a decrease in 4-NTP SERS signal when the periodicity was decreased from 500

$181 \mathrm{~nm}$ to $425 \mathrm{~nm}$, which was related to the dependence of the plasmonic bands on periodicity. For

182 instance, from $500 \mathrm{~nm}$ to $425 \mathrm{~nm}$, the plasmonic band is blue-shifted to the wavelengths below

$183600 \mathrm{~nm}$ in both simulations and experiment (Figures 2a and 2c).

\section{SERS mapping of hot spots on 3D plasmonic nanosensors}


185 In order to evaluate the detection limit of our 3D nanosensors, platforms were functionalized 186 with a 100 attomolar (aM) solution of the 4-NTP. The transmission optical image of the array of

187 P500 3D nanosensors is shown in Figure 5a. The SERS mapping was performed on the selected

188 area in red shown in Figure 5a. The map was generated by integrating the intensity of the 189 stretching mode of the nitro group $\left(v \mathrm{~s} \mathrm{NO}_{2}\right)$ in the $1282-1400 \mathrm{~cm}^{-1}$ spectral range. A strong 190 SERS intensity (bright regions) corresponded to the location of the 3D nanosensors, and 191 therefore, the location of the hot spots. This is the case for spot 1 in Figure 5b. However, regions 192 away from the 3D nanosensors (labelled spot 2 in Figure 5b) show a considerably weaker SERS 193 signal. This lack of enhancement was attributed to these positions containing only flat Au. As 194 shown in the inset of Figure 5a, the relationship between the location of the 3D nanosensors in 195 the optical image and the locations of strong SERS intensity was maintained. As a result, the 196 detection of 4-NTP drop casted onto the 3D nanosensors was possible even at a concentration of $197100 \mathrm{aM}$, and with a rapid acquisition time of 1 second. The distinguishable locations of the 3D 198 nanosensors based on the Raman map proved the reproducible ability of the sensors to generate a 199 strong signal for low concentrations of molecules trapped in the nanoscale hot spots. 200 Noteworthy, as stated previously, the engineered 3D nanosensors allow a generation of coupled 201 SPP and LSPR, where generates strong hot spots between nanoholes and nanocones. In the 202 meantime, the 3D structure of nanosensors potentially increases the surface area for attachment 203 of probe molecules to the surface of the nanostructure compared to a planar structure. This effect 204 has been observed in other studies for SERS substrates compared to a 2D array of nanosensors. ${ }^{38}$ 205 For instance, in this case, the 3D nanosensors have surface area on both top and bottom surfaces 206 of gold NHA membrane as well as on the nanocone itself compared to the planar NHA structure. 
209 In order to evaluate the limit of detection of such cavity-based sensors, the platforms were

210 functionalized with 4-NTP at concentrations between $1 \mu \mathrm{M}$ to $1 \mathrm{aM}$. In order to avoid cross

211 contamination between high and low concentration experiments, each SERS experiment was

212 repeated 3-5 times onto freshly prepared arrays of 3D nanosensors (P500-P425). Furthermore,

213 the experiments were conducted for an average of 10-15 spots on each platform. Figure 6a shows

214 that all the main peaks of the 4-NTP were detectable between $1 \mu \mathrm{M}$ to $100 \mathrm{aM} \cdot{ }^{44}$ However, we

215 observed a small but measurable change in the intensity of the SERS signal for 1aM 4-NTP,

216 even though at this concentration, it was statistically unlikely to find a spot with a single or a few

217 molecules trapped in the plasmonic cavity of the 3D nanosensors. Compared to higher

218 concentrations of 4-NTP, there were fewer spots on the 3D nanosensors that provided SERS

219 signal and the signals were not stable over long exposures. In most of the 2D plasmonic

220 substrates for SERS, providing a reproducible global signal requires at least a homogeneous

221 monolayer of the probe molecule attached onto the surface. ${ }^{38}$ The advantage of nanostructures

222 with an embedded cavity can be highlighted here as they provide a better opportunity to trap the

223 molecule in the nanoscale hot spots compared to equivalent 2D structures. Reliable Raman signal

224 collected from these 3D nanosensors was obtained for concentrations down to 100 aM. This can

225 be clearly observed by evaluating the intensity of the main peak of $\mathrm{NO}_{2}$ (Figure $6 \mathrm{a}$ inset).

226 Comparing the signal at 100 and $1 \mathrm{aM}$, it is apparent that the signal has mostly vanished for 1

227 aM. These measurements yield a limit of detection between 1-100 aM (Figure 6a).

228 Due to the fact that altering the conditions of the experiment plays a key role in obtaining the

229 SERS signal for different structures, the measurements for 100 aM 4-NTP were repeated with 
230 microscope objectives of different numerical apertures (N.A.). Increasing the N.A. of the 231 objective resulted in an enhanced SERS signal (compare the main peak of $\mathrm{NO}_{2}$ in Figure 6b).

232 The SERS signals were enhanced almost two times when increasing the N.A. from 0.5 to 0.75 233 and three times when increasing the N.A. up to 0.9.

234 By increasing the N.A. of the microscope objective, the laser beam was more confined at the 235 apex of the nanocone and the bottom of the nanohole resulting in a more efficient hot spot and 236 LSPR. It can also be beneficial to decrease the laser spot even further to be more focused on the

237 3D nanosensors, thereby excluding the scattering from flat Au regions around the hot spots. To 238 evaluate the sensitivity of different 3D nanosensors based on their periodicities, the SERS signals

239 have been collected with the same concentration of 100 aM. As shown in Figure 6c, a 240 decremental trend is observed for the SERS signal when the periodicity of the platforms was 241 decreased from 500 to $425 \mathrm{~nm}$. These results have the similar trend as compared to 242 measurements performed with $1 \mathrm{mM}$ 4-NTP. This trend was clearly observed, as shown in the 243 inset of Figure 6c.

\section{Estimation of a SERS enhancement factor}

245 Generally speaking the definition of the SERS EF can be considered as the ratio between the 246 SERS intensity per adsorbed molecule and the normal Raman intensity per bulk molecule.

247 However, in SERS, the EF for a given molecule varies with the opto-geometric conditions of the 248 SERS measurement and corresponding reference measurement. ${ }^{45}$ The determination of the 249 number of molecules that yield the Raman signal and their contribution to the EF is not trivial 250 and may lead to erroneous estimations. The ensemble of parameters that need to be considered 251 when performing a SERS experiment, such as probing a single molecule or multiple molecules, 
252 the orientation of the molecules in the experimental system, the spatial distribution, or the

253 experimental limitations in resolution, can only be used to approximate an EF.

254 The Raman signal is enhanced through both the excitation and the emission processes as shown 255 in Equation 2:46

$$
F=\left|\left(E_{v_{\text {excitation }}}\right)\right|^{2}\left|\left(E_{v_{\text {Raman }}}\right)\right|^{2}
$$

257 where $E\left(v_{\text {excitation }}\right)$ and $E\left(v_{\text {Raman }}\right)$ are the local electric-field $E F s$ at the incident frequency 258 (vexcitation) and at the Raman Stokes frequency (vRaman), respectively. However, since the plasmon 259 frequency width is large compared to the Raman Stokes shift; both electromagnetic fields are 260 often in resonance and/or pre-resonance with the plasmon band. Therefore, an approximation is 261 to assume that $E($ vexcitation $)$ and $E\left(v_{\text {Raman }}\right)$ are the same, leading to an $E F$ proportional to $F=$ $262 \mid\left. E($ vexcitation $)\right|^{4}$. In such an approximation, the matching of the excitation laser line with the 263 plasmon frequency is an essential condition to obtain large Raman surface enhancements. In 264 order to establish the relationship between the nanostructured surface and the SERS activity of 265 the platform, the EFs have been determined. The determination of the EF in SERS is a 266 prerequisite to quantify the enhancement of the Raman signal. The surface Raman EF can be 267 estimated by comparing the measured SERS intensities (ISERS) with the nonenhanced Raman 268 scattering intensities $\left(I_{\mathrm{NE}}\right)$ as shown in Equation 3:47-48

$$
E F=\frac{N_{N E} \times I_{S E R S}}{I_{N E} \times N_{S E R S}}=1.6 \times 10^{6}
$$

270 The EF obtained in Equation 3 is calculated based on the number of molecules that can be placed 271 in the focal volume of the confocal laser spot and their generated surface-enhanced-Raman 272 signals. The Raman intensity of the main peak of 4-NTP, stretching mode of the nitro group (vs 
$273 \mathrm{NO}_{2}$ ), was used as the reference peak for these calculations. Further details regarding the

274 approximations and calculations can be found in SI. Such EF obtained for 100 aM solution in a

275 femtoliter plasmonic cavity represents the promising chemical sensitivity of these 3D 276 nanosensors.

\section{CONCLUSIONS}

278 In this work, for the first time, the capability of cavity-based plasmonic nanosensors is 279 demonstrated for SERS-based molecular sensing. These nanosensors provide extraordinary 280 optical transmission properties, which can generate strong SPP and LSPR coupling. These 281 phenomena have been shown here by simulated and experimental optical transmission 282 measurements. The experimental results were also in good agreement with the FDTD 283 calculations for electromagnetic field distributions at the plasmonic bands of the nanosensors. In

284 the meantime, these structures represent a plasmonic tunability with respect to the media of the 285 experiment and also the wavelength of the incident light. These 3D nanosensors also provide a 286 remarkable molecular limit of detection between 1-100 aM for a probe molecule (4-NTP) with a 287 short acquisition time of 1 second. Consequently, a reliable EF of $10^{6}$ is achieved for these 288 sensors for an extreme low concentration of $100 \mathrm{aM}$ from a femtoliter plasmonic probe volume. 289 Reproducible SERS signals have been collected on the 3D nanosensors with concentrations 290 down to 100 aM providing the spatial distribution of hot spots on the plasmonic substrate. It is 291 also shown that by increasing the N.A. of the objectives, an enhancement occurs for the SERS 292 signals obtained on these sensors proving the confined strong LSPR coupling in the NHA-NCA 293 interface. The strong LSPR coupling of these nanosensors can introduce them to other 294 spectroscopic techniques such as SEF, SEIRA, and even tip-enhanced Raman spectroscopy 
295 (TERS) in which there is better control over the polarization of light. Of even greater interest, the

296 plasmonic tunability of these sensors can be used for simultaneous optical trapping and surface-

297 enhanced detection.

298 METHODS

\section{Fabrication of 3D plasmonic cavity nanosensors}

300 The plasmonic cavities were fabricated using electron beam lithography (EBL) methodology.

301 First, electron-beam physical vapor deposition (EB-PVD) was used to deposit a $3 \mathrm{~nm}$ thin Ti

302 layer on a Pyrex substrate. This ensured that the substrate surface was conductive for the EBL

303 writing process. A $500 \mathrm{~nm}$ thick layer of photo-resist (negative tone photoresist ma-N 2403) was

304 then spin-coated onto the Ti layer and soft baked at $90^{\circ} \mathrm{C}$ for $60 \mathrm{~s}$. The sample was placed into an

305 EBL machine (LEO, 1530 e-beam lithography) where the nanohole array patterns were written

306 on the photoresist layer. The sample was developed in MF 319 developer (Shipley, Marlborough,

307 MA) for $40 \mathrm{~s}$ leaving behind photo-resist nano-pillars, which acted as a mask to create the nano-

308 holes in the metal film. Another $3 \mathrm{~nm}$ thick Ti layer was deposited to create an adhesion layer

309 followed by $80 \mathrm{~nm}$ deposition of Au using EB-PVD deposition instrument. Once the Au layer

310 was deposited, the sample was left in PG Remover solution at $80^{\circ} \mathrm{C}$ to lift-off the photoresist

311 nano-pillars and leave behind the NHAs in the Au film. Once the NHAs were created, a TFT Ti

312 etchant (Transene Company, Inc.) was used to etch away both Ti layer and Pyrex, forming a

313 large cavity beneath the gold NHA. The sample was in Ti etchant for $70 \mathrm{~s}$ and resulted in a 250

$314 \mathrm{~nm}$ deep cavity. Afterwards, $150 \mathrm{~nm} \mathrm{Au}$ was deposited onto the structure to create a truncated

315 nano-cone beneath each nanohole on the bottom surface of the cavity. The SEM images of the

316 fabricated 3D plasmonic nanosensors with $500 \mathrm{~nm}$ periodicy are shown in Fig. 1(c). The 
317 presence of the $250 \mathrm{~nm}$ deep cavity and $150 \mathrm{~nm}$ tall truncated NCA beneath NHA the membrane

318 structure are shown in these SEM images. Each 3D nanosensor had a dimension of

319 approximately $5 \mu \mathrm{m}$ by $5 \mu \mathrm{m}$ and was repeated in a 7 by 7 square lattice arrangement with a

320 periodicity of $10 \mu \mathrm{m}$. In order to clean the platform for further use, $\mathrm{O}_{2}$ plasma or $\mathrm{UV}-\mathrm{O}_{3}$

321 exposure are efficient methods. The substrate can also be cleaned by an acid solution (Nanostrip)

322 to remove all the impurities and subsequently washed with ultrapure MilliQ water and dried

323 under nitrogen prior to $\mathrm{O}_{2}$ or $\mathrm{UV}-\mathrm{O}_{3}$ cleaning.

\section{Numerical simulation of 3D plasmonic cavity nanosensors}

325 Modeling of the electromagnetic field was performed using finite-difference time-domain 326 (FDTD) method to numerically solve Maxwell's equations (FDTD Solutions, Lumerical Inc.,

327 Vancouver, Canada). As shown Figure SI.1b, FDTD calculations were performed by creating a

328 3D unit cell that was simulated with periodic boundary conditions on the $\mathrm{x}$ - and $\mathrm{y}$-axes, and a 329 perfect match layer (PML) boundary condition in z axis. A rectangular, Cartesian style mesh was 330 placed around the unit cell with a maximum mesh setting of $3 \mathrm{~nm}$. The mesh settings can be

331 altered to increase the accuracy of the simulation by increasing the number of points within the 332 mesh.

\section{Optical characterization setup}

334 The extinction spectra of the platforms were measured, using an inverted microscope (Nikon, 335 TE300) attached to a photometer (PTI, D104), monochromator (PTI, 101), and a photo336 multiplier (PTI, 710). A $100 \mathrm{~W}$ halogen lamp produced unpolarized white light, which was 337 focused onto the structure using a bright-field condenser lens (N.A. $=0.3$ ) on the microscope. A 
338 X20 objective (N.A. $=0.45$; Nikon, 93150) was used to collect the scattered light, which was

339 then guided to the photometer using a beam splitter. Light from a desired region on the sample

340 was selected by adjusting the apertures on the photometer. The light from this desired region was

341 then guided to the monochromator for spectral analysis. The optical transmission spectra were

342 corrected for the background intensity (dark noise) and normalized by the intensity of the light

343 source.

\section{SERS measurements and sample preparation}

345 The Raman measurements were performed using a Horiba Jobin-Yvon Raman spectrometer

346 equipped with a $600 \mathrm{~g} / \mathrm{mm}$ grating and a 632.8 or $785 \mathrm{~nm}$ excitation with proper interference and

347 edge filters. For both laser sources, intensities were set to $2 \mathrm{~mW}$ or $200 \mu \mathrm{W}$ at the sample using

348 neutral density filters with 1.0 or 2.0 optical densities, respectively. Olympus microscope

349 objectives of X20 (N.A.= 0.5), X40 (N.A.=0.75), and X100 (N.A.=0.9) were used for all

350 experiments. The pinhole of the spectrometer was opened to $200 \mu \mathrm{m}$. All of the Raman spectra

351 collected for individual spots were the result of $3 \mathrm{~s}$ exposures; while the maps were the result of

$3521 \mathrm{~s}$ exposures. The maps were integrated within 1316 to $1354 \mathrm{~cm}^{-1}$. A stock solution of 4-NTP

$353\left(10^{-3} \mathrm{M}\right)$ in ethanol was made. This stock solution was then further diluted to yield $2 \mathrm{~mL}$ of

354 solutions with concentrations ranging from $10^{-6}$ to $10^{-18} \mathrm{M}$. Two drops of the as-prepared

355 solution $(\sim 100 \mu \mathrm{L})$ were deposited onto one platform, and then placed into a petri-dish. All

356 glassware used for functionalization and washing were new to avoid contamination. The petri-

357 dish was sealed, and stored in the refrigerator for the duration of the functionalization. For the

358 determination of limit of detection, the platforms were functionalized overnight (24 hours). Each

359 platform was then washed into a beaker of ethanol (99.9\%) 3 times to remove any excess of 4- 
360 NTP not adsorbed onto the surface. The platforms were then dried under nitrogen. Each SERS

361 measurement was performed 3-5 times. The spectra of 10-15 spots were collected on each 3D

362 nanosensor and the average value of the intensities was used in all relevant graphs (Figure SI4).

363 ASSOCIATED CONTENT

364 SUPPORTING INFORMATION AVAILABLE: Layout of FDTD simulation and schematic

365 representation of the 3D nanosensors, SERS signals of P475, and P450 for $1 \mathrm{mM}$ 4-NTP with

366 different conditions, simulated optical transmission spectra of 3D cavity plasmonic nanosensors

367 in ethanol, detailed EF calculations, and standard error analysis of SERS signals are shown. This

368 material is available free of charge via the Internet at http://pubs.acs.org.

369 AUTHOR INFORMATION

370 Corresponding Authors

371 *E-mail: flagugne@uwo.ca (F.L.-L.); jcarson@lawsonimaging.ca (J. J. L. C.)

372 Notes

373 The authors declare no competing financial interest.

\section{ACKNOWLEDGMENT}

375 The authors wish to gratefully acknowledge the Center for Advanced Material and Biomaterial

376 for supporting the work done in the Nanofabrication Facility at The University of Western

377 Ontario. This research was funded by Natural Sciences and Engineering Research Council of

378 Canada (NSERC) Discovery Grants (F.L.-L., J.J.L.C., B.K.) and by the Canada Research Chairs 
program (F.L.-L., B.K.). Dr. Mohamadreza Najiminaini was partially supported by the MITACS

380 program.

\section{REFERENCES}

382 1. Brolo, A. G., Plasmonics for future biosensors. Nat. Photon. 2012, 6, 709-713.

383 2. Passarelli, N.; Pérez, L. A.; Coronado, E. A., Plasmonic Interactions: From Molecular 384 Plasmonics and Fano Resonances to Ferroplasmons. ACS Nano 2014, 8, 9723-9728.

385 3. Sonntag, M. D.; Klingsporn, J. M.; Zrimsek, A. B.; Sharma, B.; Ruvuna, L. K.; Van 386 Duyne, R. P., Molecular plasmonics for nanoscale spectroscopy. Chem. Soc. Rev. 2014, 43, 387 1230-1247.

388 4. Baffou, G.; Quidant, R., Nanoplasmonics for chemistry. Chem. Soc. Rev. 2014, 43, 38983893907.

390 5. Fleischmann, M.; Hendra, P. J.; McQuillan, A. J., Raman spectra of pyridine adsorbed at 391 a silver electrode. J. Chem. Phys. Lett. 1974, 26, 163-166.

392 6. Jeanmaire, D. L.; Van Duyne, R. P., Surface raman spectroelectrochemistry: Part I. 393 Heterocyclic, aromatic, and aliphatic amines adsorbed on the anodized silver electrode. $J$. 394 Electroanal. Chem. 19977, 84, 1-20.

3957 7 Nie, S.; Emory, S. R., Probing Single Molecules and Single Nanoparticles by Surface396 Enhanced Raman Scattering. Science 1997, 275, 1102-1106.

397 8. Kneipp, K.; Wang, Y.; Kneipp, H.; Perelman, L. T.; Itzkan, I.; Dasari, R. R.; Feld, M. S., 398 Single Molecule Detection Using Surface-Enhanced Raman Scattering (SERS). Phys. Rev. Lett. 399 1997, 78, 1667-1670.

$4009 . \quad$ Sheehan, P. E.; Whitman, L. J., Detection Limits for Nanoscale Biosensors. Nano Lett. 401 2005, 5, 803-807.

402 10. Squires, T. M.; Messinger, R. J.; Manalis, S. R., Making it stick: convection, reaction and 403 diffusion in surface-based biosensors. Nat. Biotech. 2008, 26, 417-426.

404 11. Kumar, S.; Cherukulappurath, S.; Johnson, T. W.; Oh, S.-H., Millimeter-Sized 405 Suspended Plasmonic Nanohole Arrays for Surface-Tension-Driven Flow-Through SERS. 406 Chem. Mater. 2014, 26, 6523-6530.

407 12. Brolo, A. G.; Arctander, E.; Gordon, R.; Leathem, B.; Kavanagh, K. L., Nanohole408 Enhanced Raman Scattering. Nano Lett. 2004, 4, 2015-2018.

409 13. Im, H.; Sutherland, J. N.; Maynard, J. A.; Oh, S.-H., Nanohole-Based Surface Plasmon 410 Resonance Instruments with Improved Spectral Resolution Quantify a Broad Range of 411 Antibody-Ligand Binding Kinetics. Anal. Chem. 2012, 84, 1941-1947.

412 14. Barnes, W. L.; Dereux, A.; Ebbesen, T. W., Surface plasmon subwavelength optics. 413 Nature 2003, 424, 824-830.

414 15. Ebbesen, T. W.; Lezec, H. J.; Ghaemi, H. F.; Thio, T.; Wolff, P. A., Extraordinary optical 415 transmission through sub-wavelength hole arrays. Nature 1998, 391, 667-669.

416 16. Valsecchi, C.; Brolo, A. G., Periodic Metallic Nanostructures as Plasmonic Chemical 417 Sensors. Langmuir 2013, 29, 5638-5649.

418 17. Lee, M.; Kim, J.; Seo, W.; Hong, H.-G.; Song, Y.; Dasari, R. R.; An, K., Three419 dimensional imaging of cavity vacuum with single atoms localized by a nanohole array. Nat. 420 Commun. 2014, 5. 
18. Krishnan, A.; Thio, T.; Kim, T. J.; Lezec, H. J.; Ebbesen, T. W.; Wolff, P. A.; Pendry, J.; Martin-Moreno, L.; Garcia-Vidal, F. J., Evanescently coupled resonance in surface plasmon enhanced transmission. Optics Commun. 2001, 200, 1-7. 19. Najiminaini, M.; Vasefi, F.; Kaminska, B.; Carson, J. J. L., Effect of surface plasmon energy matching on the sensing capability of metallic nano-hole arrays. Appl. Phys. Lett. 2012, $100,063110$.

20. Najiminaini, M.; Vasefi, F.; Kaminska, B.; Carson, J. J. L., Nano-hole array structure with improved surface plasmon energy matching characteristics. Appl. Phys. Lett. 2012, 100, 043105 .

21. Przybilla, F.; Degiron, A.; Laluet, J.-Y.; Genet, C.; Ebbesen, T. W., Optical transmission in perforated noble and transition metal films. J. Opt. A: Pure Appl. Opt. 2006, 8, 458.

22. Brolo, A. G.; Gordon, R.; Leathem, B.; Kavanagh, K. L., Surface Plasmon Sensor Based on the Enhanced Light Transmission through Arrays of Nanoholes in Gold Films. Langmuir 2004, 20, 4813-4815.

23. Najiminaini, M.; Ertorer, E.; Kaminska, B.; Mittler, S.; Carson, J. J. L., Surface plasmon resonance sensing properties of a 3D nanostructure consisting of aligned nanohole and nanocone arrays. Analyst 2014, 139, 1876-1882.

24. Gordon, R.; Sinton, D.; Kavanagh, K. L.; Brolo, A. G., A New Generation of Sensors Based on Extraordinary Optical Transmission. Acc. Chem. Res. 2008, 41, 1049-1057.

25. Im, H.; Wittenberg, N. J.; Lesuffleur, A.; Lindquist, N. C.; Oh, S.-H., Membrane protein biosensing with plasmonic nanopore arrays and pore-spanning lipid membranes. Chem. Sci. 2010, $1,688-696$.

26. Im, H.; Lee, S. H.; Wittenberg, N. J.; Johnson, T. W.; Lindquist, N. C.; Nagpal, P.; Norris, D. J.; Oh, S.-H., Template-Stripped Smooth Ag Nanohole Arrays with Silica Shells for Surface Plasmon Resonance Biosensing. ACS Nano 2011, 5, 6244-6253.

27. Wang, Y.; Kar, A.; Paterson, A.; Kourentzi, K.; Le, H.; Ruchhoeft, P.; Willson, R.; Bao, J., Transmissive Nanohole Arrays for Massively-Parallel Optical Biosensing. ACS Photon. 2014, $1,241-245$.

28. Ricciardi, A.; Consales, M.; Quero, G.; Crescitelli, A.; Esposito, E.; Cusano, A., Versatile Optical Fiber Nanoprobes: From Plasmonic Biosensors to Polarization-Sensitive Devices. ACS Photon. 2014, 1, 69-78.

29. Genet, C.; Ebbesen, T. W., Light in tiny holes. Nature 2007, 445, 39-46.

30. Yokogawa, S.; Burgos, S. P.; Atwater, H. A., Plasmonic Color Filters for CMOS Image Sensor Applications. Nano Lett. 2012, 12, 4349-4354.

31. Yanik, A. A.; Cetin, A. E.; Huang, M.; Artar, A.; Mousavi, S. H.; Khanikaev, A.; Connor, J. H.; Shvets, G.; Altug, H., Seeing protein monolayers with naked eye through plasmonic Fano resonances. Proc. Nat. Acad. Sci. 2011, 108, 11784-11789.

32. Cervantes Tellez, G. A.; Hassan, S. a.; Tait, R. N.; Berini, P.; Gordon, R., Atomically flat symmetric elliptical nanohole arrays in a gold film for ultrasensitive refractive index sensing. Lab Chip 2013, 13, 2541-2546.

33. Fayyaz, S.; Tabatabaei, M.; Hou, R.; Lagugné-Labarthet, F., Surface-Enhanced Fluorescence: Mapping Individual Hot Spots in Silica-Protected 2D Gold Nanotriangle Arrays. J. Phys. Chem. C 2012, 116, 11665-11670.

34. Aroca, R. F.; Ross, D. J.; Domingo, C., Surface-Enhanced Infrared Spectroscopy. Appl. Spectrosc. 2004, 58, 324A-338A. 
35. Jensen, T.; Kelly, L.; Lazarides, A.; Schatz, G., Electrodynamics of Noble Metal Nanoparticles and Nanoparticle Clusters. J. Cluster Sci. 1999, 10, 295-317.

36. Merlen, A.; Lagugné-Labarthet, F., Imaging the Optical Near Field in Plasmonic Nanostructures. Appl. Spectrosc. 2014, 68, 1307-1326.

$470 \quad 37 . \quad$ Bukasov, R.; Ali, T. A.; Nordlander, P.; Shumaker-Parry, J. S., Probing the Plasmonic Near-Field of Gold Nanocrescent Antennas. ACS Nano 2010, 4, 6639-6650. 38. Tabatabaei, M.; Sangar, A.; Kazemi-Zanjani, N.; Torchio, P.; Merlen, A.; LagugnéLabarthet, F., Optical Properties of Silver and Gold Tetrahedral Nanopyramid Arrays Prepared by Nanosphere Lithography. J. Phys. Chem. C 2013, 117, 14778-14786.

39. Brown, L. V.; Yang, X.; Zhao, K.; Zheng, B. Y.; Nordlander, P.; Halas, N. J., FanShaped Gold Nanoantennas above Reflective Substrates for Surface-Enhanced Infrared Absorption (SEIRA). Nano Lett. 2015, 15, 1272-1280. Surface-Enhanced Raman Scattering on Gold Nanohole and Nanodisk Arrays. Nano Lett. 2008, 8, 1923-1928.

41. Zheng, P.; Cushing, S. K.; Suri, S.; Wu, N., Tailoring plasmonic properties of gold nanohole arrays for surface-enhanced Raman scattering. Phys. Chem. Chem. Phys. 2015. 42. Palik, E. D., Handbook of Optical Constants of Solids, Academic Press, New York, 1985. 43. Thio, T.; Ghaemi, H. F.; Lezec, H. J.; Wolff, P. A.; Ebbesen, T. W., Surface-plasmonenhanced transmission through hole arrays in Cr films. J. Opt. Soc. Am. B 1999, 16, 1743-1748. 44. Skadtchenko, B. O.; Aroca, R., Surface-enhanced Raman scattering of p-nitrothiophenol: Molecular vibrations of its silver salt and the surface complex formed on silver islands and colloids. Spectrochim. Acta A Mol. Biomol. Spectrosc. 2001, 57, 1009-1016. 45. Marquestaut, N.; Martin, A.; Talaga, D.; Servant, L.; Ravaine, S.; Reculusa, S.; Bassani, D. M.; Gillies, E.; Lagugné-Labarthet, F., Raman Enhancement of Azobenzene Monolayers on Substrates Prepared by Langmuir-Blodgett Deposition and Electron-Beam Lithography Techniques. Langmuir 2008, 24, 11313-11321.

46. Schatz, G. C.; Young, M. A.; Van Duyne, R. P. In Surface-Enhanced Raman Scattering; Kneipp, K., Moskovits, M.,Kneipp, H., Eds.; Springer-Verlag Berlin: Berlin/Heidelberg, 2006; Vol. 103, pp 19-46.

47. Félidj, N.; Truong, S. L.; Aubard, J.; Lévi, G.; Krenn, J. R.; Hohenau, A.; Leitner, A.; Aussenegg, F. R., Gold particle interaction in regular arrays probed by surface enhanced Raman scattering. J. Chem. Phys. 2004, 120, 7141-7146.

48. Félidj, N.; Aubard, J.; Lévi, G.; Krenn, J. R.; Salerno, M.; Schider, G.; Lamprecht, B.; 

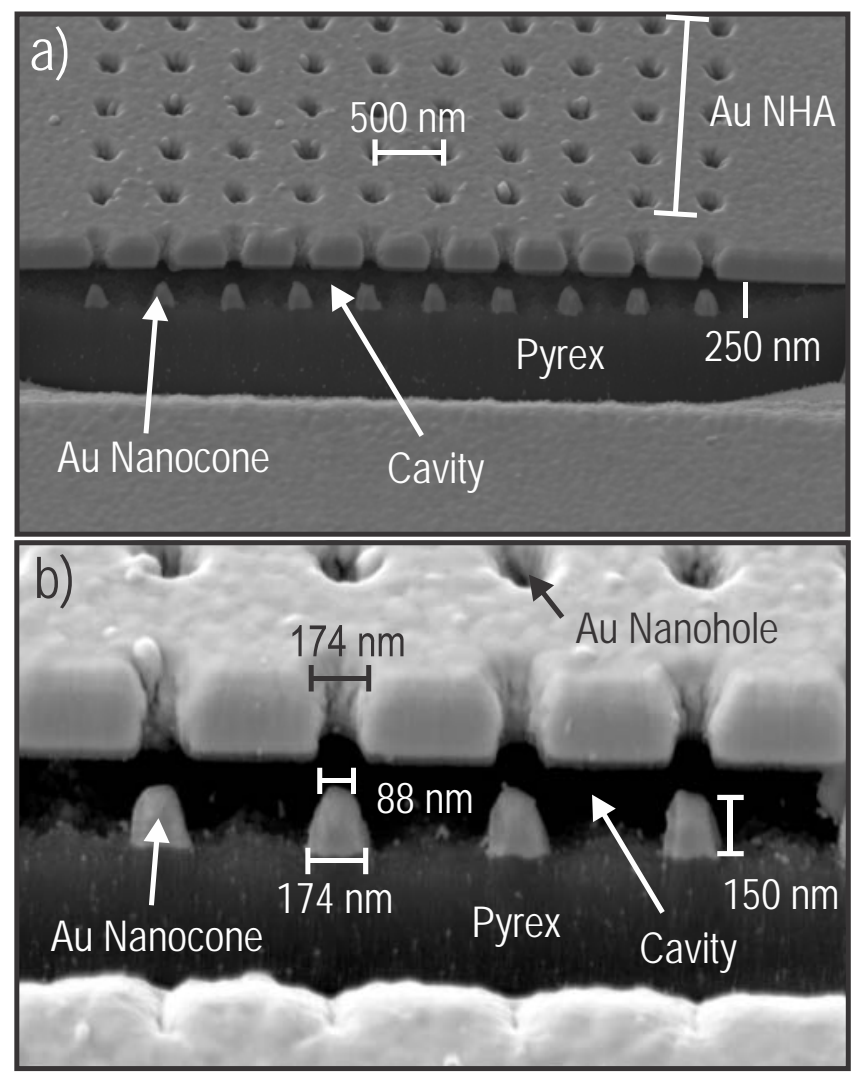

507 Figure 1. SEM images of 3D plasmonic cavity nanosensors composed of a NHA membrane with 508 co-registered NCA. a) A $230 \mathrm{~nm}$ thick Au NHA membrane with $500 \mathrm{~nm}$ periodicity and $87 \mathrm{~nm}$ 509 hole radius fabricated on a Pyrex substrate with a single $250 \mathrm{~nm}$ deep cavity. b) Magnified image 510 shown in (a) representing the dimensions of the truncated Au nanocones with an apex radius of $51144 \mathrm{~nm}$, a base radius of $87 \mathrm{~nm}$, and a height of $150 \mathrm{~nm}$. 

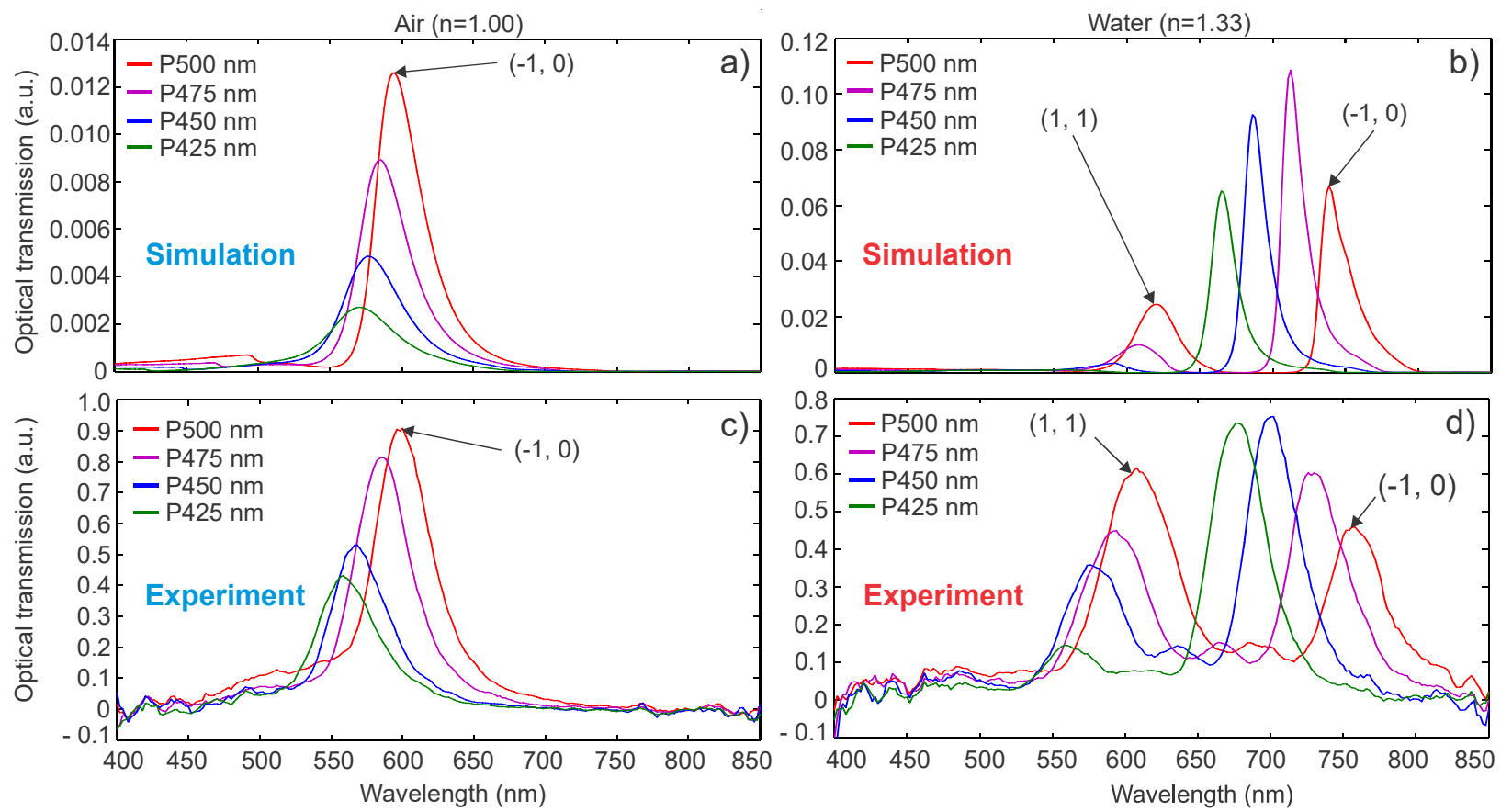

514 Figure 2. Optical transmission spectra of 3D plasmonic nanosensors for simulated and 515 experimental results. The periodicities range from $425 \mathrm{~nm}$ (green curve) to $500 \mathrm{~nm}$ (red curve) 516 with increments of $25 \mathrm{~nm}$. Simulated results for (a) air $(\mathrm{n}=1.00)$, (b) water $(\mathrm{n}=1.33)$, 517 experimental results for $(c)$ air $(n=1.00)$, and $(d)$ water $(n=1.33)$. 

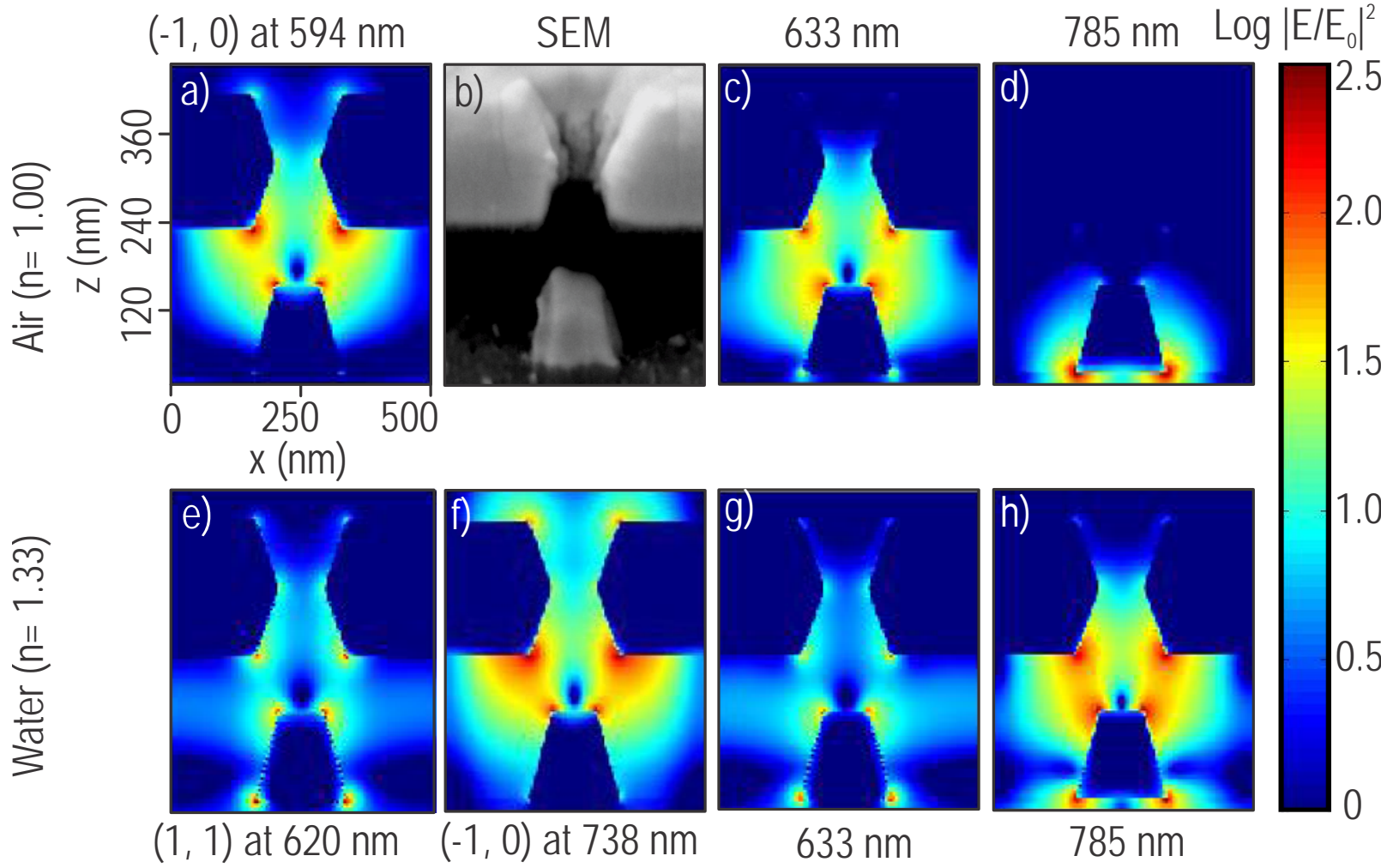

Figure 3. Electric field $\left(|\mathrm{E} / \mathrm{E} 0|^{2}, \log\right.$ scale representation) intensity of a unit cell in a 3D plasmonic nanosensor displayed on the $x z$ plane. The electric field intensity for air $(\mathrm{n}=1.00)$ at (a) $(-1,0)$ peak at $594 \mathrm{~nm}$, (c) $633 \mathrm{~nm}$, and (d) $780 \mathrm{~nm}$. The SEM image of the actual structure represented simulated images has been shown in (b). The electric field intensity for water $(\mathrm{n}=$ $1.33)$ at (e) $(1,1)$ peak at $620 \mathrm{~nm}$, (f) $(-1,0)$ peak at $738 \mathrm{~nm},(\mathrm{~g}) 633 \mathrm{~nm}$, and (h) $785 \mathrm{~nm}$. 

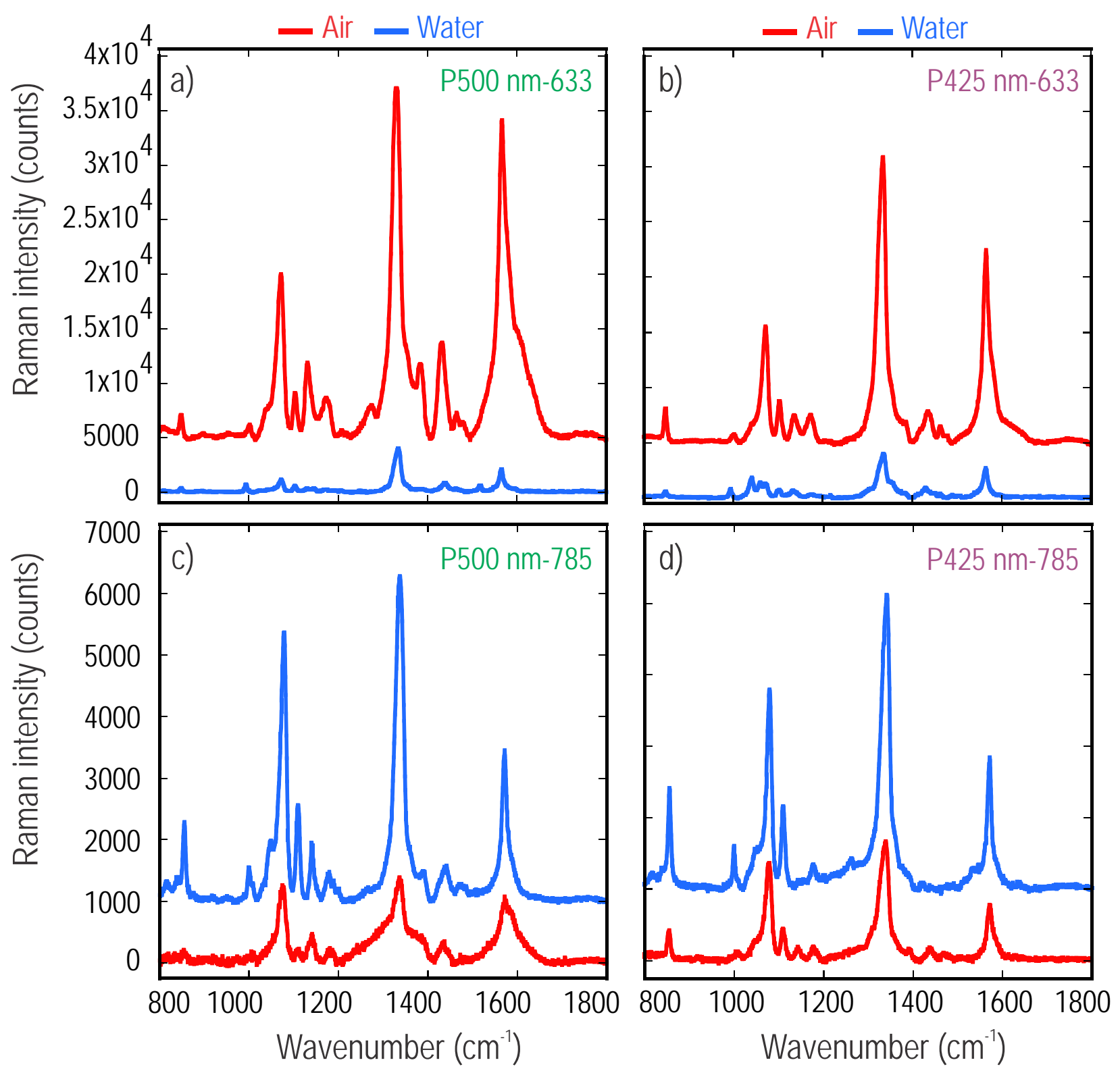

Figure 4. SERS spectra of 4-NTP adsorbed on the 3D nanosensors with different periodicities, medium (air and water) and wavelength of incident light. a) P500 nm and b) P425 nm periodicities at $633 \mathrm{~nm}$ incident light in air (red) and water (blue); c) P500 nm and d) P425 nm periodicities at $785 \mathrm{~nm}$ in air (red) and water (blue). Acquisition time for each spectrum was $3 \mathrm{~s}$ with 5 accumulations. Base line correction was applied to all spectra. A +5000 a.u. offset was applied to both red spectra in a and b. A +1000 a.u. offset was applied to both blue spectra in c and $\mathrm{d}$. These offsets were applied to represent the data in a more comparable fashion. 

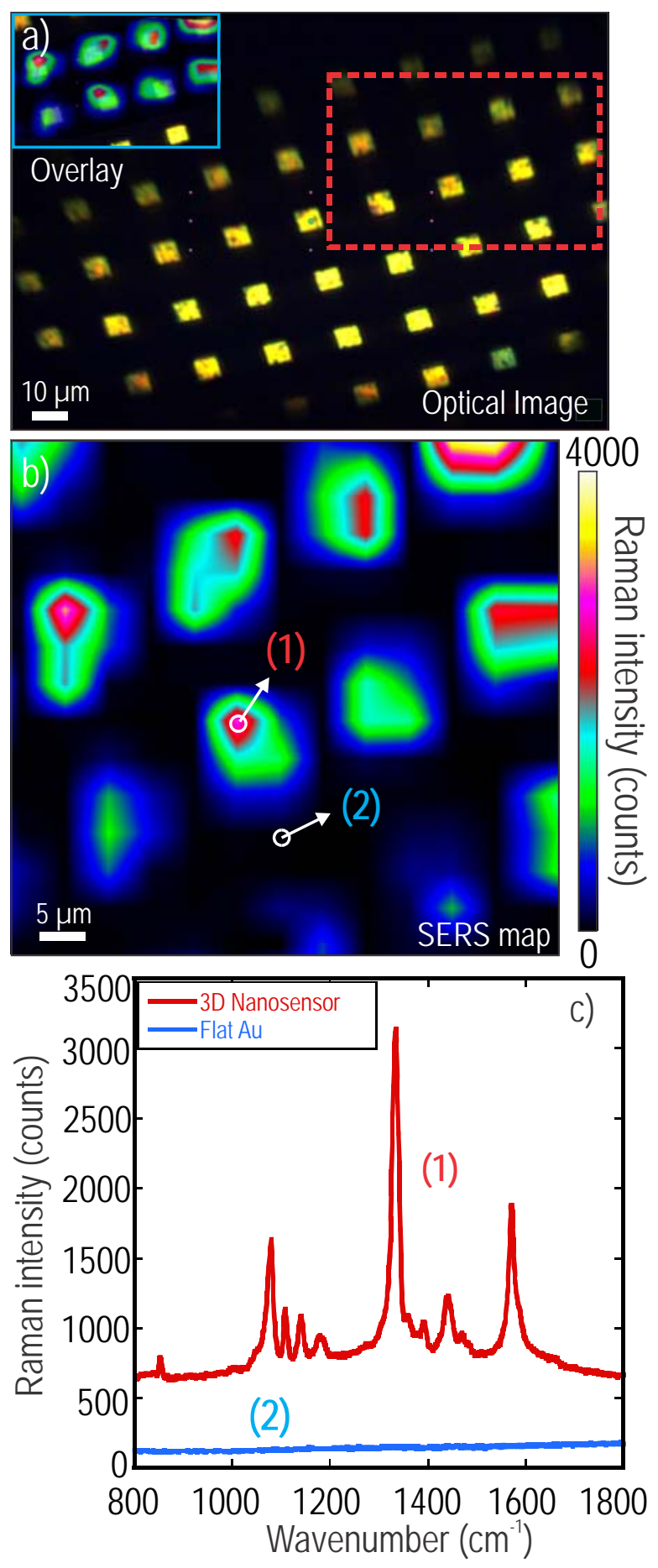

Figure 5. Surface enhanced Raman mapping of 4-NTP adsorbed on the 3D nanosensors with $500 \mathrm{~nm}$ periodicity in air with $633 \mathrm{~nm}$ incident light. Acquisition time for each spectrum was $1 \mathrm{~s}$ with $1 \mu \mathrm{m}$ step size. a) Transmission optical image of 3D nanosensors with overlaid SERS mapping (inset) for the area outlined with red dashed box. b) Raman mapping of the outlined area in panel (a). c) Spectra of the regions marked (1) and (2) in panel (b). No baseline correction was applied to spectra in panel (c). 

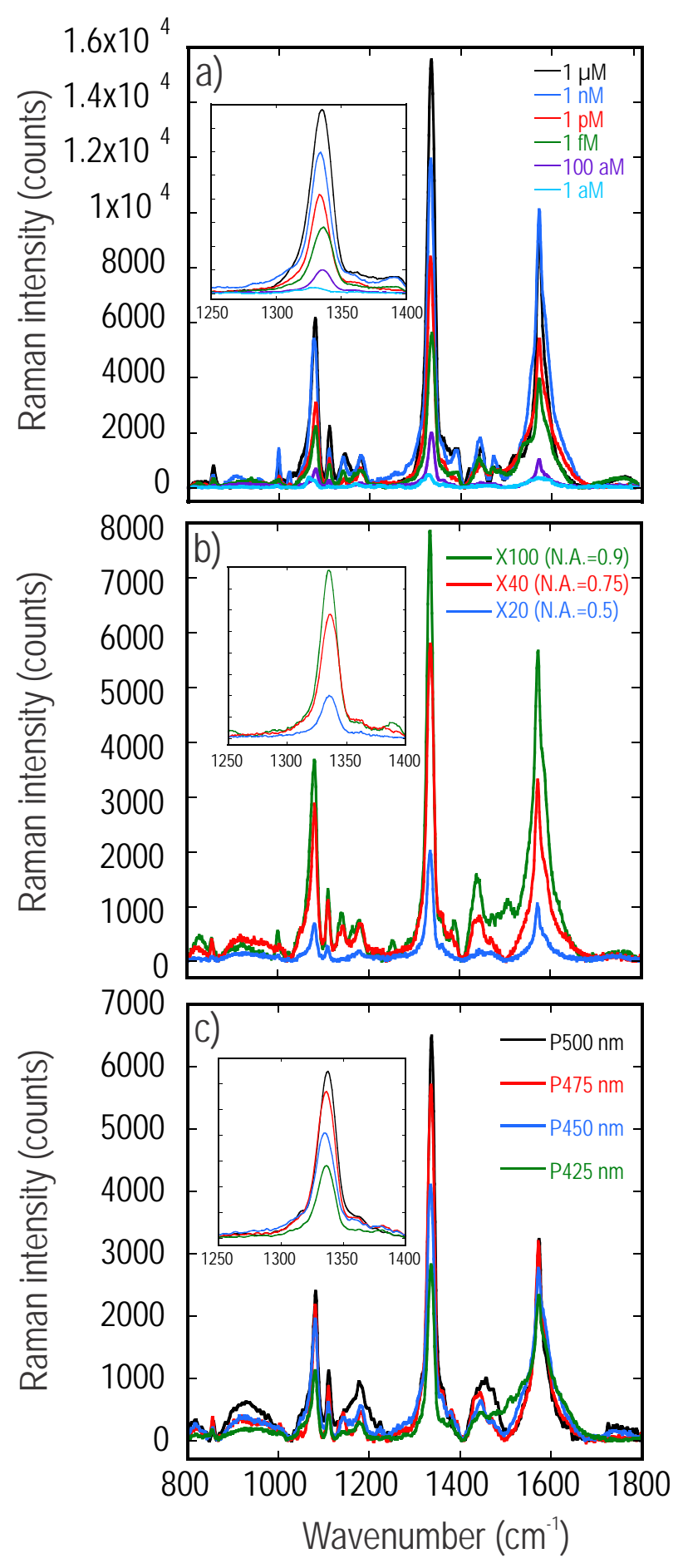

Figure 6. SERS spectra of 4-NTP adsorbed onto 3D nanosensors collected by using $633 \mathrm{~nm}$ incident laser in air. a) Different concentrations (1 aM-1 mM) of 4-NTP adsorbed on the 3D nanosensors with $500 \mathrm{~nm}$ periodicity; b) The effect of numerical aperture and magnification on SERS spectra of 100 aM 4-NTP absorbed on the 3D nanosensors; c) SERS spectra of 100 aM 4NTP adsorbed on to the 3D nanosensors. Baseline correction was applied to all spectra. SERS signals of the main $\mathrm{NO}_{2}$ peak are shown within each inset in each panel. 


\section{Table of Content Image}

Tunable 3D plasmonic cavity nanosensor for SERS

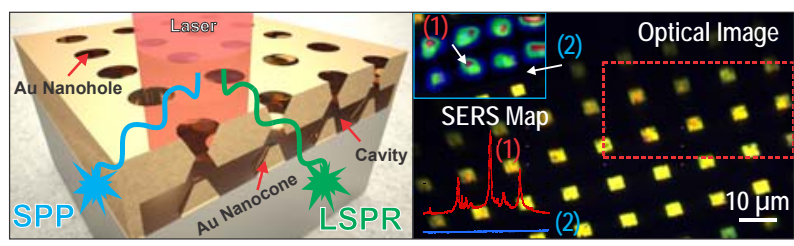

